1 Hacettepe Journal of Mathematics and Statistics

$\bigcap$ Volume $44(5)$ (2015), 1163-1179

\title{
Hom-Leibniz superalgebras and hom-Leibniz poisson superalgebras
}

\author{
Chunyue Wang*, Qingcheng Zhang ${ }^{\dagger}$ and Zhu $\mathrm{Wei}^{\ddagger}$
}

\begin{abstract}
This paper aims to characterize Hom-Leibniz superalgebras and HomLeibniz Poisson superalgebras, presents the methods to construct these superalgebras. Moreover, derivations and representations of HomLeibniz Poisson superalgebras are also investigated.
\end{abstract}

Keywords: Hom-Leibniz superalgebras, Hom-superdialgebras, Hom-Leibniz Poisson superalgebras, endomorphism.

2000 AMS Classification: 16E40,17B56,17B68,17B70.

Received 01/02/2014 : Accepted 24/07/2014 Doi : 10.15672/HJMS.2015449664

\section{Introduction}

Leibniz algebras are introduced by Cuvier and Loday [11,17], motivated by the study of algebraic $K$-theory. Such algebras are a non-antisymmetric version of Lie algebras. Active investigations on Leibniz algebras show that many results of Lie algebras can be extended to Leibniz algebras [1,5-7,18-19]. Leibniz superalgebras, originally were introduced by Dzhumadil'daev in [12], can be seen as a direct generalization of Leibniz algebras. Some theories of superdialgebras and (co)homology of Leibniz superalgebras are investigated [14-16].

During the past decades, there is an increasing interest in exploring some exotic algebraic structures [9-10]. In particular, Casas and Datuashoili considered algebras with brackets [8]. Such algebras are called noncommutative Leibniz Poisson algebras. On the other hand the dual algebraic operads of the classical operads provide some kinds of algebraic structures: Dialgebras, Dendriform algebras and Trialgebras [20].

Recently, Leibniz algebras are generalized to Hom-Leibniz algebras by Makhlouf and Silvestrov in [21]. Some structure theories of Hom-Leibniz algebras are developed [22].

*School of Applied Sciences, Jilin Teachers' Institute of Engineering and Technology, Changchun, China.

Email: wang1chun2yue3@163.com

†School of Mathematics and Statistics, Northeast Normal University, Changchun, China. Email:zhangqc569@nenu.edu.cn Corresponding Author.

${ }^{\ddagger}$ School of Mathematics and Statistics, Northeast Normal University, Changchun, China. Email:weiz986@nenu.edu.cn 
Moreover, the dialgebras are also generalized to Hom-dialgebras by Yau in [26], which give rise to Hom-Leibniz algebras. Hom-Lie algebras, Hom-Lie superalgebras and Hom-Lie color algebras have been widely investigated [13,25,3,4,2,27,23-24]. The purpose of this paper is to introduce and study Hom-Leibniz superalgebras and Hom-Leibniz Poisson superalgebras.

The paper is organized as follows. In section 2, we give the definition and some important constructions of Hom-Leibniz superalgebras. In section 3, the notion of Homsuperdialgebras is proposed, the construction of Hom-Leibniz superalgebras is provided. Moreover, we give the definition of representation of Hom-superdialgebras and show that the representation of Hom-superdialgebras gives rise to the representation of Hom-Leibniz superalgebras via a special bracket. In section 4, we introduce the notions of Hom-Leibniz Poisson superalgebras, Hom-associative supertrialgebras and Hom-dendriform superalgebras, furthermore, construct several classes of Hom-Leibniz Poisson superalgebras. Section 5 and Section 6 are devoted to dealing with the derivations and representations of Hom-Leibniz Poisson superalgebras.

Throughout this paper, $\mathbb{K}$ denotes a field of characteristic zero. All vector spaces and algebras are $\mathbb{Z}_{2}$-graded over $\mathbb{K}$.

\section{Hom-Leibniz Superalgebras}

In this section, we introduce the notion of Hom-Leibniz superalgebras, and then give the construction of Hom-Leibniz superalgebras.

2.1. Definition. ([3]) A Hom-associative superalgebra is a triple $(V, \circ, \alpha)$ consisting of a superspace $V$, an even bilinear map $\circ: V \times V \rightarrow V$ and an even superspace homomorphism $\alpha: V \rightarrow V$ satisfying

$$
\begin{aligned}
& \alpha(x \circ y)=\alpha(x) \circ \alpha(y), \\
& \alpha(x) \circ(y \circ z)=(x \circ y) \circ \alpha(z),
\end{aligned}
$$

for all homogeneous elements $x, y, z \in V$.

2.2. Definition. ([3]) A Hom-Lie superalgebra is a triple $(V,[.],, \alpha)$ consisting of a superspace $V$, an even bilinear map $[.,]:. V \times V \rightarrow V$ and an even superspace homomorphism $\alpha: V \rightarrow V$ satisfying

$$
\begin{aligned}
& \alpha([x, y])=[\alpha(x), \alpha(y)], \\
& {[x, y]=-(-1)^{|x \| y|}[y, x],} \\
& (-1)^{|x||z|}[\alpha(x),[y, z]]+(-1)^{|z||y|}[\alpha(z),[x, y]]+(-1)^{|x||y|}[\alpha(y),[z, x]]=0,
\end{aligned}
$$

for all homogeneous elements $x, y, z \in V$.

2.3. Definition. A Hom-Leibniz superalgebra is a triple $(V,[.],, \alpha)$ consisting of a superspace $V$, an even bilinear map [., .] $: V \times V \rightarrow V$ and an even superspace homomorphism $\alpha: V \rightarrow V$ satisfying

$$
\begin{aligned}
& \alpha([x, y])=[\alpha(x), \alpha(y)], \\
& {[[x, y], \alpha(z)]=[\alpha(x),[y, z]]+(-1)^{|y||z|}[[x, z], \alpha(y)],}
\end{aligned}
$$

for all homogeneous elements $x, y, z \in V$.

Let $(V,[.,],. \alpha)$ and $\left(V^{\prime},[., .]^{\prime}, \alpha^{\prime}\right)$ be two Hom-Leibniz superalgebras. An even homomorphism $f: V \rightarrow V^{\prime}$ is said to be a morphism of Hom-Leibniz superalgebras if

$$
f \circ \alpha=\alpha^{\prime} \circ f, \quad[f(x), f(y)]^{\prime}=f([x, y]), \forall x, y \in V .
$$


2.4. Remark. We recover the classical Leibniz superalgebra when $\alpha$ is an identity map and reduces to a Hom-Leibniz algebra when the part of parity one is trivial. Obviously, a Hom-Lie superalgebra is a Hom-Leibniz superalgebra. While a Hom-Leibniz superalgebra is a Hom-Lie superalgebra if and only if $[x, x]=0$, for all homogeneous element $x \in V$.

Suppose that $(V,[.,],. \alpha)$ is a Hom-Leibniz superalgebra. For any $x \in V$, define $A d_{y} \in$ $\operatorname{End}(V)$ by

$$
A d_{y}(x)=(-1)^{|x||y|}[x, y] .
$$

Then the Hom-Leibniz superalgebra identity (0.7) is written into

$$
A d_{\alpha(z)}([x, y])=(-1)^{|x||z|}\left[\alpha(x), A d_{z}(y)\right]+\left[A d_{z}(x), \alpha(y)\right],
$$

or into pure operation form

$$
A d_{\alpha(z)} A d_{y}=A d_{A d_{z}(y)} \circ \alpha+(-1)^{|y||z|} A d_{\alpha(y)} \circ A d_{z} .
$$

The following proposition provides a method to construct a Hom-Leibniz superalgebra by a Leibniz superalgebra and an even endomorphism.

2.5. Proposition. Let $(V,[.,]$.$) be a Leibniz superalgebra and \alpha: V \rightarrow V$ be an even Leibniz superalgebra endomorphism. Then $\left(V,[., .]_{\alpha}, \alpha\right)$ is a Hom-Leibniz superalgebra, where $[x, y]_{\alpha}=\alpha([x, y])$.

Moreover, suppose that $\left(V^{\prime},[., .]^{\prime}\right)$ is another Leibniz superalgebra and $\alpha^{\prime}: V^{\prime} \rightarrow V^{\prime}$ is a Leibniz superalgebra endomorphism. If $f: V \rightarrow V^{\prime}$ is a Leibniz superalgebra morphism that satisfies $f \circ \alpha=\alpha^{\prime} \circ f$, then

$$
f:\left(V,[., .]_{\alpha}, \alpha\right) \rightarrow\left(V^{\prime},[., .]_{\alpha^{\prime}}^{\prime}, \alpha^{\prime}\right)
$$

is a morphism of Hom-Leibniz superalgebras.

Proof. We show that $\left(V,[., .]_{\alpha}, \alpha\right]$ satisfies the Hom-Leibniz superalgebra identity $(0.7)$. In fact,

$$
\begin{aligned}
& {\left[\alpha(x),[y, z]_{\alpha}\right]_{\alpha}+(-1)^{|y||z|}\left[[x, z]_{\alpha}, \alpha(y)\right]_{\alpha} } \\
= & \alpha([\alpha(x), \alpha([y, z])])+(-1)^{|y||z|} \alpha([\alpha([x, z]), \alpha(y)]) \\
= & \alpha^{2}\left([x,[y, z]]+(-1)^{|y||z|}[[x, z], y]\right) \\
= & \alpha^{2}([[x, y], z]) \\
= & {\left[[x, y]_{\alpha}, \alpha(z)\right]_{\alpha} }
\end{aligned}
$$

The second assertion follows from

$$
\begin{aligned}
f\left([x, y]_{\alpha}\right) & =f([\alpha(x), \alpha(y)]) \\
& =[f \circ \alpha(x), f \circ \alpha(y)]^{\prime} \\
& =\left[\alpha^{\prime} \circ f(x), \alpha^{\prime} \circ f(y)\right]^{\prime} \\
& =[f(x), f(y)]_{\alpha^{\prime}}^{\prime} .
\end{aligned}
$$

2.6. Example. (3-dimensional Hom-Leibniz superalgebras) Let $A=A_{\overline{0}} \oplus A_{\overline{1}}$ be a 3 -dimensional superspace, where $A_{\overline{0}}$ is generated by $e_{1}$ and $A_{\overline{1}}$ is generated by $e_{2}, e_{3}$ and the nonzero product is given by $\left[e_{2}, e_{1}\right]=e_{2}$. For any $a, b \in \mathbb{K}$, we consider the homomorphism $\alpha: A \rightarrow A$ defined by $\alpha\left(e_{1}\right)=a e_{1}, \alpha\left(e_{3}\right)=b e_{2}$. By Proposition 2.5, for any $a \in \mathbb{K}$, there is the corresponding Hom-Leibniz superalgebra $A_{\alpha}=\left(A,[., .]_{\alpha}, \alpha\right)$ with 
the nonzero product $\left[e_{2}, e_{1}\right]_{\alpha}=a e_{2}$. It is not a Leibniz superalgebra when $a \neq 0,1$.

2.7. Lemma. Let $V$ be a Hom-Lie superalgebra, then the bracket

$$
[x \otimes y, a \otimes b]=[[x, y], \alpha(a)] \otimes b+(-1)^{|a||x|+|a||y|} a \otimes[[x, y], \alpha(b)]
$$

definies a Hom-Leibniz superalgebra structure on the vector superspace $V \otimes V$.

2.8. Definition. A representation (module) of the Hom-Leibniz superalgebra $(V,[.,],. \alpha)$ is a Hom-supermodule $\left(U, \alpha_{U}\right)$ equipped with two even $V$-actions (left and right)

$$
[., .]: U \times V \rightarrow U \quad((u, x) \mapsto[u, x]) \text { and }[., .]: V \times U \rightarrow U \quad((x, u) \mapsto[x, u])
$$

satisfying the following axioms,

$$
\begin{aligned}
& {\left[U_{\alpha}, V_{\beta}\right] \subseteq U_{\alpha+\beta}, \forall \alpha, \beta \in \mathbb{Z}_{2},} \\
& {\left[V_{\alpha}, U_{\beta}\right] \subseteq U_{\alpha+\beta}, \forall \alpha, \beta \in \mathbb{Z}_{2},} \\
& \alpha_{U}([u, x])=\left[\alpha_{U}(u), \alpha(x)\right], \\
& \alpha_{U}([x, u])=\left[\alpha(x), \alpha_{U}(u)\right], \\
& {[[u, x], \alpha(y)]=\left[\alpha_{U}(u),[x, y]\right]+(-1)^{|x||y|}[[u, y], \alpha(x)],} \\
& {[[x, u], \alpha(y)]=[\alpha(x),[u, y]]+(-1)^{|u||y|}\left[[x, y], \alpha_{U}(u)\right],}
\end{aligned}
$$

for all homogeneous elements $x, y \in V$ and $u \in U$.

Note that the last two relations imply the following identity

$$
[\alpha(x),[u, y]]+(-1)^{|u||y|}[\alpha(x),[y, u]]=0 .
$$

\section{Hom-Superdialgebras}

In this section, we extend in one hand superdialgebras and the Hom-dialgebras introduced in [14] and [26] to Hom-superdialgebras. In the other hand we describe some constructions of Hom-Leibniz superalgebras.

3.1. Definition. ([14]) A superdialgebra is a triple $(V, \dashv, \vdash)$ consisting of a superspace $V$, two even bilinear maps $\dashv, \vdash: V \times V \rightarrow V$ satisfying

$$
\begin{aligned}
& (0.20) \quad x \vdash(y \dashv z)=(x \vdash y) \dashv z, \\
& (0.21) \quad x \dashv(y \dashv z)=(x \dashv y) \dashv z=x \dashv(y \vdash z), \\
& (0.22) \quad x \vdash(y \vdash z)=(x \vdash y) \vdash z=(x \dashv y) \vdash z,
\end{aligned}
$$

for all homogeneous elements $x, y, z \in V$.

3.2. Definition. A Hom-superdialgebra is a tuple $(V, \dashv, \vdash, \alpha)$ consisting of a superspace $V$, two even bilinear maps $\dashv, \vdash: V \times V \rightarrow V$ and an even superspace homomorphism $\alpha: V \rightarrow V$ satisfying

$$
\alpha(x \dashv y)=\alpha(x) \dashv \alpha(y), \quad \alpha(x \vdash y)=\alpha(x) \vdash \alpha(y),
$$$$
\alpha(x) \dashv(y \dashv z)=(x \dashv y) \dashv \alpha(z)=\alpha(x) \dashv(y \vdash z),
$$$$
\alpha(x) \vdash(y \vdash z)=(x \vdash y) \vdash \alpha(z)=(x \dashv y) \vdash \alpha(z),
$$

$$
\alpha(x) \vdash(y \dashv z)=(x \vdash y) \dashv \alpha(z),
$$

for all homogeneous elements $x, y, z \in V$.

3.3. Remark. We recover the classical superdialgebra [14] when $\alpha$ is an identity 
map and reduces to a Hom-dialgebra [26] when the part of parity one is trivial. Any Hom-associative superalgebra is a Hom-superdialgebra if $a \vdash b=a \dashv b=a b$.

3.4. Proposition. If $\left(V_{1}, \circ_{1}, \alpha_{1}\right)$ and $\left(V_{2}, \circ_{2}, \alpha_{2}\right)$ are two Hom-superdialgebras, then the tensor product $V_{1} \otimes V_{2}$ is a Hom-superdialgebra with

$$
\alpha=\alpha_{1} \otimes \alpha_{2},
$$

and

$$
\left(v_{1} \otimes v_{2}\right) \star\left(u_{1} \otimes u_{2}\right)=(-1)^{\left|v_{2}\right|\left|u_{1}\right|}\left(v_{1} \star u_{1}\right) \otimes\left(v_{2} \star u_{2}\right)
$$

for all homogeneous elements $v_{1}, v_{2} \in V_{1}, u_{1}, u_{2} \in V_{2}$ and $\star=\dashv, \quad \vdash$.

3.5. Definition. Let $(V, \dashv, \vdash, \alpha)$ and $\left(V^{\prime}, \dashv^{\prime}, \vdash^{\prime}, \alpha^{\prime}\right)$ be two Hom-superdialgebras. An even homomorphism $f: V \rightarrow V^{\prime}$ is said to be a morphism of Hom-superdialgebras if $f \circ \alpha=\alpha^{\prime} \circ f$, and $f(x) \dashv^{\prime} f(y)=f(x \dashv y)$, and $f(x) \vdash^{\prime} f(y)=f(x \vdash y)$ for any $x, y \in V$.

3.6. Proposition. Let $(V, \dashv, \vdash)$ be a superdialgebra and $\alpha: V \rightarrow V$ be an even superdialgebra endomorphism. Then $\left(V, \dashv_{\alpha}, \vdash_{\alpha}, \alpha\right)$ is a Hom-superdialgebra, where $x \dashv_{\alpha} y=$ $\alpha(x \dashv y)$ and $x \vdash_{\alpha} y=\alpha(x \vdash y)$.

Moreover, suppose that $\left(V^{\prime}, \dashv^{\prime}, \vdash^{\prime}\right)$ is another superdialgebra and $\alpha^{\prime}: V^{\prime} \rightarrow V^{\prime}$ is a superdialgebra endomorphism. If $f: V \rightarrow V^{\prime}$ is a superdialgebra morphism that satisfies $f \circ \alpha=\alpha^{\prime} \circ f$, then

$$
f:\left(V, \dashv_{\alpha}, \vdash_{\alpha}, \alpha\right) \rightarrow\left(V^{\prime}, \dashv^{\prime}{ }_{\alpha^{\prime}}, \vdash^{\prime}{ }_{\alpha^{\prime}}, \alpha^{\prime}\right)
$$

is a morphism of Hom-superdialgebras.

Proof. We only need to show that $\left(V, \dashv_{\alpha}, \vdash_{\alpha}, \alpha\right)$ satisfies the Hom-superdialgebra identity (0.24)-(0.26). Direct calculations show that

$$
\begin{aligned}
\alpha(x) \dashv_{\alpha}\left(y \vdash_{\alpha} z\right) & =\alpha(\alpha(x) \dashv \alpha(y \dashv z)) \\
& =\alpha^{2}(x \dashv(y \dashv z)) \\
& =\alpha^{2}((x \dashv y) \dashv z) \\
& =\left(x \dashv_{\alpha} y\right) \dashv_{\alpha} \alpha(z),
\end{aligned}
$$

and

$$
\begin{aligned}
\alpha(x) \dashv_{\alpha}\left(y \vdash_{\alpha} z\right) & =\alpha^{2}(x \dashv(y \dashv z)) \\
& =\alpha^{2}(x \dashv(y \vdash z)) \\
& =\alpha\left(\alpha(x) \dashv\left(y \vdash_{\alpha} z\right)\right) \\
& =\alpha(x) \dashv_{\alpha}\left(y \vdash_{\alpha} z\right),
\end{aligned}
$$

thus (0.24) holds. Similarly, we can prove (0.25) and (0.26).

Setting $\star_{\alpha}=\dashv_{\alpha}$ and $\star_{\alpha}=\vdash_{\alpha}$. The second assertion follows from

$$
f \circ \star_{\alpha}=f \circ \alpha \circ \star=\alpha^{\prime} \circ f \circ \star=\alpha^{\prime} \circ \star^{\prime} \circ f=\star_{\alpha^{\prime}} \circ f .
$$

3.7. Proposition. Let $(V, \dashv, \vdash, \alpha)$ be a Hom-superdialgebra. Define an even bilinear $\operatorname{map}[.,]:. V \times V \rightarrow V$ by

$$
[x, y]=x \dashv y-(-1)^{|x||y|} y \vdash x, \forall x, y \in V .
$$

Then $(V,[.,],. \alpha)$ is a Hom-Leibniz superalgebra. 
Proof. We only need to show that $(V,[.,],. \alpha)$ satisfies the Hom-Leibniz superalgebra identity (0.7). Direct calculations show that

$$
\begin{aligned}
& {[\alpha(x),[y, z]]+(-1)^{|y||z|}[[x, z], \alpha(y)] } \\
= & \alpha(x) \dashv(y \dashv z)-(-1)^{|x||y|+|x||z|}(y \dashv z) \vdash \alpha(x) \\
- & (-1)^{|y||z|} \alpha(x) \dashv(z \vdash y)+(-1)^{|x||y|+|x||z|+|y||z|}(z \vdash y) \vdash \alpha(x) \\
+ & (-1)^{|y||z|}(x \dashv z) \dashv \alpha(y)-(-1)^{|x||y|} \alpha(y) \vdash(x \dashv z) \\
- & (-1)^{|x||z|+|y||z|}(z \vdash x) \dashv \alpha(y)+(-1)^{|x||y|+|x||z|} \alpha(y) \vdash(z \vdash x) \\
= & (x \dashv y) \dashv \alpha(z)-(-1)^{|x||z|+|y| \mid z} \alpha(z) \vdash(x \dashv y) \\
- & (-1)^{|x||y|}(y \vdash x) \dashv \alpha(z)+(-1)^{|x||y|+|x||| z|+| y|| z \mid} \alpha(z) \vdash(y \vdash x) \\
+ & (-1)^{|y||z|}\{(x \dashv z) \dashv \alpha(y)-\alpha(x) \dashv(z \vdash y)\} \\
+ & (-1)^{|x||y|+|x||z|}\{\alpha(y) \vdash(z \vdash x)-(y \dashv z) \vdash \alpha(x)\} \\
= & {[x \dashv y, \alpha(z)]-(-1)^{|x||y|}[y \vdash x, \alpha(z)] } \\
= & {[[x, y], \alpha(z)] . }
\end{aligned}
$$

3.8. Proposition. Let $\left(V,[.,],. \alpha_{1}\right)$ be a Hom-Leibniz superalgebra, $\left(U, \dashv, \vdash, \alpha_{2}\right)$ be a super commutative Hom-superdialgebra and let $g=V \otimes U$. Define the operations $\alpha: g \rightarrow g$ and $[.,]:. g^{\otimes 2} \rightarrow g$ by

(0.29) $\alpha=\alpha_{1} \otimes \alpha_{2}$,

$(0.30) \quad[x \otimes a, y \otimes b]=(-1)^{|a||y|}[x, y] \otimes(a \vdash b)$.

Then $(g,[.,],. \alpha)$ is a Hom-Leibniz superalgebra.

Proof. We only need to show that $(g,[.,],. \alpha)$ satisfies the Hom-Leibniz superalgebra identity (0.7). Direct calculations show that

$$
\begin{aligned}
& {[\alpha(x \otimes a),[y \otimes b, z \otimes c]]+(-1)^{|y||z|+|y||c|+|b||z|+|b||c|}[[x \otimes a, z \otimes c], \alpha(y \otimes b)] } \\
= & {\left[\alpha_{1}(x) \otimes \alpha_{2}(a),(-1)^{|b||z|}[y, z] \otimes(b \vdash c)\right] } \\
+ & (-1)^{|y||z|+|y||c|+|b||z|+|b||c|+|a||z|}\left[[x, z] \otimes(a \vdash c), \alpha_{1}(y) \otimes \alpha_{2}(b)\right] \\
= & (-1)^{|a||y|+|a||z|+|b||z|}\left[\alpha_{1}(x),[y, z]\right] \otimes\left(\alpha_{2}(a) \vdash(b \vdash c)\right) \\
+ & (-1)^{|a||y|+|a||z|+|b||z|+|b||c|+|y||z|}\left[[x, z], \alpha_{1}(y)\right] \otimes\left((a \vdash c) \vdash \alpha_{2}(b)\right) \\
= & (-1)^{|a||y|+|a||z|+|b||z|}\left[\alpha_{1}(x),[y, z]\right] \otimes\left(\alpha_{2}(a) \vdash(b \vdash c)\right) \\
+ & (-1)^{|a||y|+|a||z|+|b||z|+|y||z|}\left[[x, z], \alpha_{1}(y)\right] \otimes\left(\alpha_{2}(a) \vdash(b \vdash c)\right) \\
= & (-1)^{|a||y|+|a||z|+|b||z|}\left\{\left[\alpha_{1}(x),[y, z]\right]\right. \\
+ & \left.(-1)^{|y||z|}\left[[x, z], \alpha_{1}(y)\right]\right\} \otimes\left(\alpha_{2}(a) \vdash(b \vdash c)\right) \\
= & (-1)^{|a||y|+|a||z|+|b||z|}\left[[x, y], \alpha_{1}(z)\right] \otimes\left(\alpha_{2}(a) \vdash(b \vdash c)\right) .
\end{aligned}
$$

and

$$
\begin{aligned}
& {[[x \otimes a, y \otimes b], \alpha(z \otimes c)]=\left[(-1)^{|a||y|}[x, y] \otimes(a \vdash b), \alpha_{1}(z) \otimes \alpha_{2}(c)\right] } \\
= & (-1)^{|a||y|+|a||z|+|b||z|}\left[[x, y], \alpha_{1}(z)\right] \otimes\left((a \vdash b) \vdash \alpha_{2}(c)\right) \\
= & (-1)^{|a||y|+|a||z|+|b||z|}\left[[x, y], \alpha_{1}(z)\right] \otimes\left(\alpha_{2}(a) \vdash(b \vdash c)\right) .
\end{aligned}
$$


This shows that $(g,[.,],. \alpha)$ is a Hom-Leibniz superalgebra.

3.9. Definition. Let $(V, \dashv, \vdash, \alpha)$ be a Hom-superdialgebra and $\left(U, \alpha_{U}\right)$ be a Homsuperspace. The pair $\left(U, \alpha_{U}\right)$ is said to be a $V$-supermodule if $\left(U, \alpha_{U}\right)$ is a Hom-supermodule equipped with four actions (left and right) of $V$

$$
\begin{aligned}
& V \otimes U \rightarrow U \quad(x \otimes u \mapsto x \dashv u \quad \text { or } \quad x \vdash u), \\
& U \otimes V \rightarrow U \quad(u \otimes x \mapsto u \dashv x \quad \text { or } \quad u \vdash x)
\end{aligned}
$$

satisfying the following axioms

$$
\begin{aligned}
\alpha_{U}(x \dashv u) & =\alpha(x) \dashv \alpha_{U}(u), \\
\alpha_{U}(x \vdash u) & =\alpha(x) \vdash \alpha_{U}(u), \\
\alpha_{U}(u \dashv x) & =\alpha_{U}(u) \dashv \alpha(x), \\
\alpha_{U}(u \vdash x) & =\alpha_{U}(u) \vdash \alpha(x), \\
\alpha(x) \dashv(y \dashv u) & =(x \dashv y) \dashv \alpha_{U}(u)=\alpha(x) \dashv(y \vdash u), \\
(x \vdash y) \dashv \alpha_{U}(u) & =\alpha(x) \vdash(y \dashv u), \\
(x \dashv y) \vdash \alpha_{U}(u) & =\alpha(x) \vdash(y \vdash u)=(x \vdash y) \vdash \alpha_{U}(u), \\
\alpha(x) \dashv(u \dashv y) & =(x \dashv u) \dashv \alpha(y)=\alpha(x) \dashv(u \vdash y), \\
(x \vdash u) \dashv \alpha(y) & =\alpha(x) \vdash(u \dashv y), \\
(x \dashv u) \vdash \alpha(y) & =\alpha(x) \vdash(u \vdash y)=(x \vdash u) \vdash \alpha(y), \\
\alpha_{U}(u) \dashv(x \dashv y) & =(u \dashv x) \dashv \alpha(y)=\alpha(u) \dashv(x \vdash y), \\
(u \vdash x) \dashv \alpha(y) & =\alpha_{U}(u) \vdash(x \dashv y), \\
(u \dashv x) \vdash \alpha(y) & =\alpha_{U}(u) \vdash(x \vdash y)=(u \vdash x) \vdash \alpha(y),
\end{aligned}
$$

for all $x, y \in V$ and $u \in U$.

3.10. Proposition. Let $(V, \dashv, \vdash, \alpha)$ be a Hom-superdialgebra, $(V,[.,],. \alpha)$ be a HomLeibniz superalgebra, where $[x, y]=x \dashv y-(-1)^{|x||y|} y \vdash x$ for any $x, y \in V$, and $\left(U, \alpha_{U}\right)$ be a representation of $(V, \dashv, \vdash, \alpha)$. Then $\left(U, \alpha_{U}\right)$ is also a representation of $(V,[.,],. \alpha)$.

Proof. We just check

$$
\left[[x, y], \alpha_{U}(u)\right]=[\alpha(x),[y, u]]+(-1)^{|y||u|}[[x, u], \alpha(y)] .
$$

Using the axioms of the supermodule of Hom-superdialgebra, we have

$$
\begin{aligned}
& {[\alpha(x),[y, u]]+(-1)^{|y||u|}[[x, u], \alpha(y)] } \\
= & \alpha(x) \dashv(y \dashv u)-(-1)^{|x||y|+|x||u|}((y \dashv u) \vdash \alpha(x)) \\
- & (-1)^{|y||u|} \alpha(x) \dashv(u \vdash y)+(-1)^{|x||y|+|x||u|+|y||u|}(u \vdash y) \vdash \alpha(x) \\
+ & (-1)^{|y||u|}(x \dashv u) \dashv \alpha(y)-(-1)^{|x||y|} \alpha(y) \vdash(x \dashv u) \\
- & (-1)^{|x||u|+|y||u|}(u \vdash x) \dashv \alpha(y)+(-1)^{|x||y|+|x||u|} \alpha(y) \vdash(u \vdash x) \\
= & (x \dashv y) \dashv \alpha_{U}(u)-(-1)^{|x||u|+|y||u|} \alpha_{U}(u) \vdash(x \dashv y) \\
- & (-1)^{|x||y|}(y \vdash x) \vdash \alpha_{U}(u)+(-1)^{|x||y|+|y||u|+|x||u|} \alpha_{U}(u) \vdash(y \vdash x) \\
= & {\left[[x, y], \alpha_{U}(u)\right] . }
\end{aligned}
$$

\section{Hom-Leibniz Poisson Superalgebras}


In this section, we introduce the notions of Hom-Leibniz Poisson superalgebras, Homassociative supertrialgebras and Hom-dendriform superalgebras. Moreover, we construct several classes of Hom-Leibniz Poisson superalgebras.

4.1. Definition. A Hom-Poisson superalgebra is a tuple $(A, \circ,[.,],. \alpha)$ consisting of a superspace $V$, two even bilinear maps $\circ,[.,]:. V \times V \rightarrow V$ and an even superspace homomorphism $\alpha: V \rightarrow V$ satisfying the following axioms

(1) $(A, \circ, \alpha)$ is a Hom-associative superalgebra,

(2) $(A,[.,],. \alpha)$ is a Hom-Lie superalgebra,

(3) the Hom-Leibniz superidentity

$$
[x \circ y, \alpha(z)]=\alpha(x) \circ[y, z]+(-1)^{|y||z|}[x, z] \circ \alpha(y)
$$

holds, for all homogeneous elements $x, y, z \in A$.

4.2. Theorem. Let $(A, \cdot,[.,]$.$) be a Poisson superalgebra and \alpha: A \rightarrow A$ be an even Poisson superalgebra endomorphism. Then $\left(A, \cdot{ }_{\alpha},[.,]_{\alpha}, \alpha\right)$ is a Hom-Poisson superalgebra, where $x \cdot{ }_{\alpha} y=\alpha(x \cdot y)$ and $[x, y]_{\alpha}=\alpha([x, y])$.

Proof. It is straightforward.

This theorem provides a method to construct Hom-Poisson superalgebra by a Poisson superalgebra and an even Poisson superalgebra endomorphism.

4.3. Example. Let $A=A_{\overline{0}} \oplus A_{\overline{1}}$ be a 2-dimensional superspace, where $A_{\overline{0}}$ is generated by $e_{1}$ and $A_{\overline{1}}$ is generated by $e_{2}$ and nonzero products are given by

$$
e_{1} \cdot e_{1}=e_{1}, \quad e_{2} \cdot e_{2}=e_{1}, \quad e_{1} \cdot e_{2}=e_{2} \cdot e_{1}=e_{2}, \quad\left[e_{2}, e_{2}\right]=2 e_{1} .
$$

For any $a \in \mathbb{K}$, we consider the homomorphism $\alpha: A \rightarrow A$ defined by

$$
\alpha\left(e_{1}\right)=a e_{1}, \quad \alpha\left(e_{2}\right)=a e_{2} .
$$

By Theorem 4.2, for any $a \in \mathbb{K}$, there is the corresponding Hom-Poisson superalgebra $A_{a}=\left(A, \cdot \alpha,[., .]_{\alpha}, \alpha\right)$ with the nonzero products

$$
e_{1} \cdot \alpha e_{1}=a e_{1}, \quad e_{2} \cdot \alpha e_{2}=a e_{1}, \quad e_{1} \cdot{ }_{\alpha} e_{\alpha}=a e_{2}, \quad\left[e_{2}, e_{2}\right]_{\alpha}=2 a e_{1} .
$$

It is not a Poisson superalgebra when $a \neq 0,1$.

4.4. Example. Let $A=A_{\overline{0}} \oplus A_{\overline{1}}$ be a 3 -dimensional superspace, where $A_{\overline{0}}$ is generated by $e_{1}, e_{2}$ and $A_{\overline{1}}$ is generated by $e_{3}$ and the nonzero products are given by

$$
e_{1} \cdot e_{2}=e_{1}, \quad e_{2} \cdot e_{2}=e_{2}, \quad e_{3} \cdot e_{2}=e_{3}, \quad\left[e_{1}, e_{2}\right]=a e_{1} .
$$

For any $a \in \mathbb{K}$, we consider the homomorphism $\alpha: A \rightarrow A$ defined by

$$
\alpha\left(e_{1}\right)=a e_{1}, \quad \alpha\left(e_{2}\right)=e_{1}+e_{2} .
$$

By Theorem 4.2, for any $a \in \mathbb{K}$, there is the corresponding Hom-Poisson superalgebra $A_{\alpha}=\left(A, \cdot{ }_{\alpha},[., \cdot]_{\alpha}, \alpha\right)$ with the nonzero products

$$
e_{1} \cdot \alpha e_{2}=a e_{1}, \quad e_{2} \cdot \alpha e_{2}=e_{1}+e_{2}, \quad\left[e_{1}, e_{2}\right]_{\alpha}=a e_{1}
$$

It is not a Poisson superalgebra when $a \neq 0,1$.

4.5. Definition. A Hom-Leibniz Poisson superalgebra is a tuple $(V, \circ,[.,],. \alpha)$ consisting of a superspace $V$, two even bilinear maps $\circ,[.,]:. V \times V \rightarrow V$ and an even superspace homomorphism $\alpha: V \rightarrow V$ satisfying the following axioms

(1) $(V, \circ, \alpha)$ is a Hom-associative superalgebra,

(2) $(V,[.,],. \alpha)$ is a Hom-Leibniz superalgebra, 
(3) the Hom-Leibniz superidentity

$$
[x \circ y, \alpha(z)]=\alpha(x) \circ[y, z]+(-1)^{|y||z|}[x, z] \circ \alpha(y)
$$

holds, for all homogeneous elements $x, y, z \in V$.

4.6. Definition. Let $(V, \circ,[.,],. \alpha)$ and $\left(V^{\prime}, \circ^{\prime},[., .]^{\prime}, \alpha^{\prime}\right)$ be two Hom-Leibniz Poisson superalgebras. An even homomorphism $f: V \rightarrow V^{\prime}$ is said to be a morphism of HomLeibniz Poisson superalgebras if

$$
\begin{aligned}
& f \circ \alpha=\alpha^{\prime} \circ f \\
& f(x) \circ^{\prime} f(y)=f(x \circ y), \quad[f(x), f(y)]^{\prime}=f([x, y]), \quad \forall x, y \in V .
\end{aligned}
$$

4.7. Remark. Any Hom-Poisson superalgebra is a Hom-Leibniz Poisson superalgebra. Any Hom-Leibniz Poisson superalgebra $(V, \circ,[.,],. \alpha)$ is a Hom-Poisson superalgebra if and only if $[x, y]+(-1)^{|x||y|}[y, x]=0$ holds, for all homogeneous elements $x, y \in V$. If $\alpha=I d$, then a Hom-Leibniz Poisson superalgebra becomes a Leibniz-Poisson superalgebra. On the other hand, any Hom-associative superalgebra is a Hom-Leibniz Poisson superalgebra with usual bracket $[x, y]=x \circ y-(-1)^{|x||y|} y \circ x$.

4.8. Proposition. Let $(V, \dashv, \vdash, \alpha)$ be a Hom-superdialgebra and $\circ,[.,]:. V \times V \rightarrow V$ be two binary operations on $V$ defined by

$$
x \circ y=x \vdash y, \quad[x, y]=x \dashv y-(-1)^{|x||y|} y \vdash x, \forall x, y \in V .
$$

Then $(V, \circ,[.,],. \alpha)$ is a Hom-Leibniz Poisson superalgebra.

Proof. It is obvious that $(V, \circ, \alpha)$ is a Hom-associative superalgebra. Moreover, from Proposition 3.7 , it follows that $(V,[.,],. \alpha)$ is a Hom-Leibniz superalgebra. Next we show the remaining Hom-Leibniz superidentity. In fact

$$
\begin{aligned}
& \alpha(x) \circ[y, z]+(-1)^{|y||z|}[x, z] \circ \alpha(y) \\
= & \alpha(x) \vdash(y \dashv z)-(-1)^{|y||z|} \alpha(x) \vdash(z \vdash y) \\
+ & (-1)^{|y||z|}(x \dashv z) \vdash \alpha(y)-(-1)^{|x||z|+|y||z|}(z \vdash x) \vdash \alpha(y) \\
= & (x \vdash y) \dashv \alpha(z)-(-1)^{|x||z|+|y||z|} \alpha(z) \vdash(x \vdash y) \\
= & {[x \circ y, \alpha(z)] . }
\end{aligned}
$$

Taking $\alpha=I d$ in Proposition 4.8, we obtain the following result about Leibniz-Poisson superalgebras.

4.9. Corollary. Let $(V, \dashv, \vdash)$ be a superdialgebra and $\circ,[.,]:. V \times V \rightarrow V$ be two binary operations on $V$ defined by

$$
x \circ y=x \vdash y, \quad[x, y]=x \dashv y-(-1)^{|x||y|} y \vdash x, \forall x, y \in V .
$$

Then $(V, \circ,[.,]$.$) is a Leibniz-Poisson superalgebra.$

4.10. Proposition. Let $(V, \circ,[.,]$.$) be a Leibniz-Poisson superalgebra and \alpha: V \rightarrow V$ be an even Leibniz-Poisson superalgebras endomorphism. Then $\left(V, \circ_{\alpha},[., .]_{\alpha}, \alpha\right)$ is a HomLeibniz Poisson superalgebra, where $x \circ_{\alpha} y=\alpha(x \circ y)$ and $[x, y]_{\alpha}=\alpha([x, y])$.

Moreover, suppose that $\left(V^{\prime}, \circ^{\prime},[.,]^{\prime}\right)$ is another Leibniz superalgebra and $\alpha^{\prime}: V^{\prime} \rightarrow$ $V^{\prime}$ is a Leibniz superalgebras endomorphism. If $f: V \rightarrow V^{\prime}$ is a Leibniz superalgebra 
morphism that satisfies $f \circ \alpha=\alpha^{\prime} \circ f$, then

$$
f:\left(V, \circ_{\alpha},[., .]_{\alpha}, \alpha\right) \rightarrow\left(V^{\prime}, \circ_{\alpha^{\prime}},[.,,]_{\alpha^{\prime}}, \alpha^{\prime}\right)
$$

is a morphism of Hom-Leibniz superalgebras.

Proof. It is obvious that $\left(V, \circ_{\alpha}, \alpha\right)$ is a Hom-associative superalgebra. Moreover, from Proposition 2.5, we have $\left(V,[., .]_{\alpha}, \alpha\right)$ is a Hom-Leibniz superalgebra. Next we will show that the Hom-Leibniz superidentity holds. In fact

$$
\begin{aligned}
& \alpha(x) \circ_{\alpha}[y, z]_{\alpha}+(-1)^{|y||z|}[x, z]_{\alpha} \circ_{\alpha} \alpha(y) \\
= & \alpha(\alpha(x) \circ \alpha([y, z]))+(-1)^{|y||z|} \alpha(\alpha([x, z]) \circ \alpha(y)) \\
= & \alpha^{2}\left(x \circ[y, z]+(-1)^{|y||z|}[x, z] \circ y\right) \\
= & \alpha^{2}[x \circ y, z] \\
= & \alpha\left(\left[x \circ_{\alpha} y, \alpha(z)\right]\right) \\
= & {\left[x \circ_{\alpha} y, \alpha(z)\right]_{\alpha} . }
\end{aligned}
$$

By Proposition 2.5, the second assertion is straightforward.

4.11. Definition. An Hom-associative supertrialgebra is a quintuple $(V, \dashv, \vdash, \perp, \alpha)$ consisting of a superspace $V$, three even bilinear maps $-, \vdash, \perp: V \times V \rightarrow V$ and an even superspace homomorphism $\alpha: V \rightarrow V$ satisfying the following axioms

$$
\begin{aligned}
\alpha(x \dashv y) & =\alpha(x) \dashv \alpha(y), & & \alpha(x \vdash y) & =\alpha(x) \vdash \alpha(y), \\
\alpha(x \perp y) & =\alpha(x) \perp \alpha(y), & & (x \dashv y) \dashv \alpha(z) & =\alpha(x) \dashv(y \dashv z), \\
(x \dashv y) \dashv \alpha(z) & =\alpha(x) \dashv(y \vdash z), & & (x \vdash y) \dashv \alpha(z) & =\alpha(x) \vdash(y \dashv z), \\
(x \dashv y) \vdash \alpha(z) & =\alpha(x) \vdash(y \vdash z), & & (x \vdash y) \vdash \alpha(z) & =\alpha(x) \vdash(y \vdash z), \\
(x \dashv y) \dashv \alpha(z) & =\alpha(x) \dashv(y \perp z), & & (x \perp y) \dashv \alpha(z) & =\alpha(x) \perp(y \dashv z), \\
(x \dashv y) \perp \alpha(z) & =\alpha(x) \perp(y \vdash z), & & (x \vdash y) \perp \alpha(z) & =\alpha(x) \vdash(y \perp z), \\
(x \perp y) \vdash \alpha(z) & =\alpha(x) \vdash(y \vdash z), & & (x \perp y) \perp \alpha(z) & =\alpha(x) \perp(y \perp z) .
\end{aligned}
$$

4.12. Remark. We recover the classical associative trialgebra when $\alpha=I d$ and the part of parity one is trivial in $[14,20]$. The associative supertrialgebra is obtained when $\alpha=I d$. Any Hom-associative supertrialgebra gives rise to a Hom-associative superdialgebra by forgetting the operation $\perp$.

4.13. Proposition. Let $(V, \dashv, \vdash, \perp, \alpha)$ be a Hom-associative supertrialgebra and $\circ,[.,]:. V \times V \rightarrow V$ be two binary operations on $V$ defined by

$$
x \circ y=x \perp y, \quad[x, y]=x \dashv y-(-1)^{|x||y|} y \vdash x, \forall x, y \in V .
$$

Then $(V, \circ,[.,],. \alpha)$ is a Hom-Leibniz Poisson superalgebra.

Proof. It is obvious that $(V, \circ, \alpha)$ is a Hom-associative superalgebra. Moreover, from Proposition 3.7, we have $(V,[.,],. \alpha)$ is a Hom-Leibniz superalgebra. Next we will show 
that the remaining Hom-Leibniz superidentity holds. In fact,

$$
\begin{aligned}
{[\alpha(x), y \circ z]+(-1)^{|y||z|}[x \circ z, \alpha(y)] } & =\alpha(x) \perp(y \dashv z) \\
& -(-1)^{|y||z|} \alpha(x) \perp(z \vdash y) \\
& +(-1)^{|y||z|}(x \dashv z) \perp \alpha(y) \\
& -(-1)^{|x||z|+|y||z|}(z \vdash x) \perp \alpha(y) \\
& =(x \perp y) \dashv \alpha(z)-(-1)^{|x||z|+|y||z|} \alpha(z) \vdash(x \perp y) \\
& =[x \circ y, \alpha(z)] .
\end{aligned}
$$

4.14. Definition. [24] A Hom-dendriform superalgebra is a tuple $(V, \prec, \succ, \alpha)$ consisting of a superspace $V$, two even bilinear maps $\prec, \succ: V \times V \rightarrow V$ and an even superspace homomorphism $\alpha: V \rightarrow V$ satisfying the following axioms

$$
\begin{aligned}
\alpha(x<y) & =\alpha(x)<\alpha(y), \\
\alpha(x>y) & =\alpha(x)>\alpha(y), \\
(x \prec y) \prec \alpha(z) & =\alpha(x) \prec(y \prec z)+\alpha(x) \prec(y \succ z), \\
(x \succ y) \prec \alpha(z) & =\alpha(x) \succ(y \prec z), \\
(x \prec y) \succ \alpha(z)+(x \succ y) \succ \alpha(z) & =\alpha(x) \succ(y \succ z),
\end{aligned}
$$

for all homogeneous elements $x, y, z \in V$.

4.15. Lemma. Let $(V, \prec, \succ, \alpha)$ be a Hom-dendriform superalgebra, define the product on homogeneous elements by

$$
x * y=x \prec y+x \succ y .
$$

Then $(V, *, \alpha)$ is a Hom-associative superalgebra.

4.16. Proposition. Let $(V, \prec, \succ, \alpha)$ be a Hom-dendriform superalgebra. Define the products on homogeneous elements by

$$
x * y=x \prec y+x \succ y, \quad[x, y]=x * y-(-1)^{|x||y|} y * x .
$$

Then $(V, *,[.,],. \alpha)$ is a Hom-Leibniz Poisson superalgebra.

Proof. It is straightforward.

\section{Derivation of Hom-Leibniz Poisson Superalgebras}

In this section, we extend the $\alpha$-derivations of Hom-Lie algebras introduced in [25] to Hom-Leibniz Poisson superalgebras.

Let $(V, \circ,[.,],. \alpha)$ be a Hom-Leibniz Poisson superalgebra, denote by $\alpha^{k}$ the $k$-times composition of $\alpha$, i.e., $\alpha^{k}=\alpha \circ \alpha \cdots \cdots \circ \alpha$ (k-times). In particular, $\alpha^{-1}=0, \alpha^{0}=I d$, and $\alpha^{1}=\alpha$.

5.1. Definition. For any $k \geq-1$, we call $D \in(\text { EndV })_{i}$, where $i \in \mathbb{Z}_{2}$, an $\alpha^{k}$ derivation of the Hom-Leibniz Poisson superalgebra $(V, \circ,[.,],. \alpha)$ if

$$
\begin{aligned}
& \alpha \circ D=D \circ \alpha, \\
& D([x, y])=\left[D(x), \alpha^{k}(y)\right]+(-1)^{|x||D|}\left[\alpha^{k}(x), D(y)\right], \\
& D(x \circ y)=D(x) \circ \alpha^{k}(y)+(-1)^{|x||D|} \alpha^{k}(x) \circ D(y),
\end{aligned}
$$


for all homogeneous elements $x, y \in V$.

We denote by $\operatorname{Der}_{\alpha^{k}}(V)=\operatorname{Der}_{\alpha^{k}}(V)_{\overline{0}} \oplus \operatorname{Der}_{\alpha^{k}}(V)_{\overline{1}}$ the set of $\alpha^{k}$-derivations of the Hom-Leibniz Poisson superalgebra $(V, \circ,[.,],. \alpha)$, and $\operatorname{Der}(V)=\oplus_{k \geq-1} \operatorname{Der}_{\alpha^{k}}(V)$.

For any homogeneous elements $a \in V$, satisfying $\alpha(a)=a$, define $a d_{k}(a) \in \operatorname{End}(V)$ by

$$
\operatorname{ad}_{k}(a)(x)=-(-1)^{|a||x|}\left[\alpha^{k}(x), a\right], \forall x \in V .
$$

Notice that $\left|a d_{k}(a)\right|=|a|$.

5.2. Proposition. Let $(V, \circ,[.,],. \alpha)$ be a Hom-Leibniz Poisson superalgebra. Then $a d_{k}(a)$ is an $\alpha^{k+1}$-derivation, which is said to be an inner $\alpha^{k+1}$-derivation.

Proof. Direct calculations show that

$$
\begin{aligned}
&{a d_{k}(a) \circ \alpha(x)}=-(-1)^{|a||x|}\left[\alpha^{k+1}(x), a\right] \\
&=-(-1)^{|a||x|}\left[\alpha^{k+1}(x), \alpha(a)\right] \\
&=-(-1)^{|a||x|} \alpha\left(\left[\alpha^{k}(x), a\right]\right. \\
&=\alpha \circ a d_{k}(a)(x),
\end{aligned}
$$

and

$$
\begin{aligned}
a d_{k}(a)([x, y]) & =-(-1)^{|a||x|+|a||y|}\left[\alpha^{k}([x, y]), a\right] \\
& =-(-1)^{|a||x|+|a||y|}\left[\left[\alpha^{k}(x), \alpha^{k}(y)\right], \alpha(a)\right] \\
& =-(-1)^{|a||x|+|a||y|}\left[\alpha^{k+1}(x),\left[\alpha^{k}(y), a\right]\right]-(-1)^{|a||x|}\left[\left[\alpha^{k}(x), a\right], \alpha^{k+1}(y)\right] \\
& =(-1)^{|a||x|}\left[\alpha^{k+1}(x), a d_{k}(a)(y)\right]+\left[a d_{k}(a)(x), \alpha^{k+1}(y)\right],
\end{aligned}
$$

and

$$
\begin{aligned}
a d_{k}(a)(x \circ y) & =-(-1)^{|a||x|+|a||y|}\left[\alpha^{k}(x \circ y), a\right] \\
& =-(-1)^{|a||x|+|a||y|}\left[\alpha^{k}(x) \circ \alpha^{k}(y), \alpha(a)\right] \\
& \left.=-(-1)^{|a||x|+|a||y|} \alpha^{k+1}(x) \circ\left[\alpha^{k}(y), a\right]\right]-(-1)^{|a||x|}\left[\alpha^{k}(x), a\right] \circ \alpha^{k+1}(y) \\
& =(-1)^{|a||x|} \alpha^{k+1}(x) \circ a d_{k}(a)(y)+a d_{k}(a)(x) \circ \alpha^{k+1}(y) .
\end{aligned}
$$

Therefore, $\operatorname{ad}_{k}(a)$ is an $\alpha^{k+1}$-derivation.

We denote by $\operatorname{Inn}_{\alpha^{k}}(V)$ the set of inner $\alpha^{k}$-derivations, i.e.,

$$
\operatorname{Inn}_{\alpha^{k}}(V)=\left\{a d_{k}(a) \mid a \in V_{\overline{0}} \cup V_{\overline{1}}, \alpha(a)=a\right\} .
$$

For any $D \in \operatorname{Der}(V)$ and $D^{\prime} \in \operatorname{Der}(V)$, define their commutator $\left[D, D^{\prime}\right]$ as usual:

$$
\left[D, D^{\prime}\right]=D \circ D^{\prime}-(-1)^{|D|\left|D^{\prime}\right|} D^{\prime} \circ D
$$

5.3. Lemma. For any $D \in\left(\operatorname{Der}_{\alpha^{k}}(V)\right)_{i}$ and $D^{\prime} \in\left(\operatorname{Der}_{\alpha^{k}}(V)\right)_{j}$, then $\left[D, D^{\prime}\right] \in$ $\operatorname{Der}_{\alpha^{k+s}}(V)_{|D|+\left|D^{\prime}\right|}$, where $k+s \geq-1$ and $(i, j) \in \mathbb{Z}_{2}^{2}$. 
Proof. For any $x, y \in V$, we have

$$
\begin{aligned}
{\left[D, D^{\prime}\right]([x, y]) } & =D \circ D^{\prime}([x, y])-(-1)^{|D|\left|D^{\prime}\right|} D^{\prime} \circ D([x, y]) \\
& =D\left(\left[D^{\prime}(x), \alpha^{s}(y)\right]+(-1)^{\left|D^{\prime}\right||x|}\left[\alpha^{s}(x), D^{\prime}(y)\right]\right) \\
& -(-1)^{|D|\left|D^{\prime}\right|} D^{\prime}\left(\left[D(x), \alpha^{k}(y)\right]+(-1)^{|D||x|}\left[\alpha^{k}(x), D(y)\right]\right) \\
& =\left[D D^{\prime}(x), \alpha^{k+s}(y)\right]+(-1)^{|D|\left|D^{\prime}\right|+|D||x|}\left[\alpha^{k}\left(D^{\prime}(x)\right), D \alpha^{s}(y)\right] \\
& +(-1)^{\left|D^{\prime}\right||x|}\left[D \alpha^{s}(x), \alpha^{k} D^{\prime}(y)\right]+(-1)^{\left|D^{\prime}\right||x|+|D||x|}\left[\alpha^{k+s}(x), D D^{\prime}(y)\right] \\
& -(-1)^{|D|\left|D^{\prime}\right|}\left[D^{\prime} D(x), \alpha^{k+s}(y)\right]-(-1)^{\left|D^{\prime}\right||x|}\left[\alpha^{s} D(x), D^{\prime} \alpha^{k}(y)\right] \\
& -(-1)^{|D|\left|D^{\prime}\right|+|D||x|}\left[D^{\prime} \alpha^{k}(x), \alpha^{s} D(y)\right] \\
& -(-1)^{|D|\left|D^{\prime}\right|+|D||x|+\left|D^{\prime}\right||x|}\left[\alpha^{k+s}(x), D^{\prime} D(y)\right] .
\end{aligned}
$$

Since $D$ and $D^{\prime}$ satisfy $D \circ \alpha=\alpha \circ D$ and $D^{\prime} \circ \alpha=\alpha \circ D^{\prime}$, we obtain

$$
\begin{aligned}
{\left[D, D^{\prime}\right]([x, y]) } & =\left[D D^{\prime}(x)-(-1)^{|D|\left|D^{\prime}\right|} D^{\prime} D(x), \alpha^{k+s}(y)\right] \\
& +(-1)^{|D||x|+\left|D^{\prime}\right||x|}\left[\alpha^{k+s}(x), D D^{\prime}(y)-(-1)^{|D|\left|D^{\prime}\right|} D^{\prime} D(y)\right] \\
& =\left[\left[D, D^{\prime}\right](x), \alpha^{k+s}(y)\right]+(-1)^{\left|\left[D, D^{\prime}\right]\right||x|}\left[\alpha^{k+s}(x),\left[D, D^{\prime}\right](y)\right]
\end{aligned}
$$

It is not difficult to show that

$$
\begin{aligned}
{\left[D, D^{\prime}\right](x \circ y) } & =D \circ D^{\prime}(x \circ y)-(-1)^{|D|\left|D^{\prime}\right|} D^{\prime} \circ D(x \circ y) \\
& =D\left(D^{\prime}(x) \circ \alpha^{s}(y)+(-1)^{\left|D^{\prime}\right||x|} \alpha^{s}(x) \circ D^{\prime}(y)\right) \\
& -(-1)^{|D|\left|D^{\prime}\right|} D^{\prime}\left(D(x) \circ \alpha^{k}(y)+(-1)^{|D||x|} \alpha^{k}(x) \circ D(y)\right) \\
& =D D^{\prime}(x) \circ \alpha^{k+s}(y)+(-1)^{|D|\left|D^{\prime}\right|+|D||x|} \alpha^{k} D^{\prime}(x) \circ D \alpha^{s}(y) \\
& +(-1)^{\left|D^{\prime}\right||x|} D \alpha^{s}(x) \circ \alpha^{k} D^{\prime}(y)+(-1)^{\left|D^{\prime}\right||x|+|D||x|} \alpha^{k+s}(x) \circ D D^{\prime}(y) \\
& -(-1)^{|D|\left|D^{\prime}\right|} D^{\prime} D(x) \circ \alpha^{k+s}(y)-(-1)^{\left|D^{\prime}\right||x|} \alpha^{s} D(x) \circ D^{\prime} \alpha^{k}(y) \\
& -(-1)^{|D|\left|D^{\prime}\right|+|D||x|} D^{\prime} \alpha^{k}(x) \circ \alpha^{s} D(y) \\
& -(-1)^{|D|\left|D^{\prime}\right|+|D||x|+\left|D^{\prime}\right||x|} \alpha^{k+s}(x) \circ D^{\prime} D(y) \\
& =\left(D D^{\prime}-(-1)^{|D|\left|D^{\prime}\right|} D^{\prime} D\right)(x) \circ \alpha^{k+s}(y) \\
& +(-1)^{\left|\left[D, D^{\prime}\right]\right||x|}\left(D D^{\prime}-(-1)^{|D|\left|D^{\prime}\right|} D^{\prime} D\right)(y) \\
& =\left[D, D^{\prime}\right](x) \circ \alpha^{k+s}(y)+(-1)^{\left|\left[D, D^{\prime}\right]\right||x|} \alpha^{k+s}(x) \circ\left[D, D^{\prime}\right](y) .
\end{aligned}
$$

It is easy to verify that $\alpha \circ\left[D, D^{\prime}\right]=\left[D, D^{\prime}\right] \circ \alpha$, which leads to $\left[D, D^{\prime}\right] \in \operatorname{Der}_{\alpha^{k+s}}(V)_{|D|+\left|D^{\prime}\right|}$.

5.4. Remark. Obviously, we have

$$
\operatorname{Der}_{\alpha^{-1}}(V)=\{D \in \operatorname{End}(V) \mid D \circ \alpha=\alpha \circ D, D([x, y])=0, D(x \circ y)=0, \forall x, y \in V\} .
$$

Thus for any $D, D^{\prime} \in \operatorname{Der}_{\alpha^{-1}}(V)$, we have $\left[D, D^{\prime}\right] \in \operatorname{Der}_{\alpha^{-1}}(V)$.

5.5. Proposition. With the above notations, $\operatorname{Der}(V)$ is a Hom-Leibniz Poisson superalgebra, in which the bracket is given by $\left[D, D^{\prime}\right]=D D^{\prime}-(-1)^{|D|\left|D^{\prime}\right|} D^{\prime} D$ and an even endomorphism $\alpha^{\prime}$ is defined by $\alpha^{\prime}(D)=\alpha \circ D$.

\section{Representations of Hom-Leibniz Poisson Superalgebras}


Let $(V, \circ,[.,],. \alpha)$ be a Hom-Leibniz Poisson superalgebra, then $(V, \circ, \alpha)$ is a Homassociative superalgebra and $(V,[.,],. \alpha)$ a Hom-Leibniz superalgebra, so we can study $V-V$-bimodules, and the representation of Hom-Leibniz superalgebras over $V$.

6.1. Definition. Let $(V, \circ,[.,],. \alpha)$ be a Hom-Leibniz Poisson superalgebra. $A V-V$ bimodule $\left(M, \alpha_{M}\right)$ is two $\mathbb{K}$-module homomorphisms

$$
[., .]: V \otimes M \rightarrow M,[., .]: M \otimes V \rightarrow M
$$

such that the following axioms hold:

$$
\begin{aligned}
{\left[V_{\alpha}, M_{\beta}\right] \subseteq M_{\alpha+\beta}, } & {\left[M_{\alpha}, V_{\beta}\right] \subseteq M_{\alpha+\beta}, \quad \forall \alpha, \beta \in \mathbb{Z}_{2}, } \\
\alpha_{M}([v, m]) & =\left[\alpha(v), \alpha_{M}(m)\right], \\
\alpha_{M}([m, v]) & =\left[\alpha_{M}(m), \alpha(v)\right], \\
{\left[\left[v_{1}, v_{2}\right], \alpha_{M}(m)\right] } & =\left[\alpha\left(v_{1}\right),\left[v_{2}, m\right]\right]+(-1)^{\left|v_{2}\right||m|}\left[\left[v_{1}, m\right], \alpha\left(v_{2}\right)\right], \\
{\left[\left[v_{1}, m\right], \alpha\left(v_{2}\right)\right] } & =\left[\alpha\left(v_{1}\right),\left[m, v_{2}\right]\right]+(-1)^{\left|v_{2}\right||m|}\left[\left[v_{1}, v_{2}\right], \alpha_{M}(m)\right], \\
{\left[\left[m, v_{1}\right], \alpha\left(v_{2}\right)\right] } & =\left[\alpha_{M}(m),\left[v_{1}, v_{2}\right]\right]+(-1)^{\left|v_{1}\right|\left|v_{2}\right|}\left[\left[m, v_{2}\right], \alpha\left(v_{1}\right)\right], \\
{\left[v_{1} \circ m, \alpha\left(v_{2}\right)\right] } & =\alpha\left(v_{1}\right) \circ\left[m, v_{2}\right]+(-1)^{|m|\left|v_{2}\right|}\left[v_{1}, v_{2}\right] \circ \alpha_{M}(m), \\
{\left[m \circ v_{1}, \alpha\left(v_{2}\right)\right] } & =\alpha_{M}(m) \circ\left[v_{1}, v_{2}\right]+(-1)^{\left|v_{1}\right|\left|v_{2}\right|}\left[m, v_{2}\right] \circ \alpha\left(v_{1}\right), \\
{\left[v_{1} \circ v_{2}, \alpha_{M}(m)\right] } & =\alpha\left(v_{1}\right) \circ\left[v_{2}, m\right]+(-1)^{\left|v_{2}\right||m|}\left[v_{1}, m\right] \circ \alpha\left(v_{2}\right),
\end{aligned}
$$

for all homogeneous elements $m \in M, v_{1}, v_{2} \in V$.

$A$ representation over $V$ is defined by a $V-V$-bimodule $\left(M, \alpha_{M}\right)$.

6.2. Proposition. Let $\left(V_{1}, \circ_{1},[., .]_{1}, \alpha_{1}\right)$ and $\left(V_{2}, \circ_{2},[., .]_{2}, \alpha_{2}\right)$ be Hom-Leibniz Poisson superalgebras and $\varphi: V_{1} \rightarrow V_{2}$ be a morphism of Hom-Leibniz Poisson superalgebras, then $V_{2}$ is a representation over $V_{1}$ with respect to the operations

$$
\begin{aligned}
& v_{1} \cdot m=\varphi\left(v_{1}\right) \cdot m, \quad m \cdot v_{1}=m \cdot \varphi\left(v_{1}\right), \\
& {\left[v_{1}, m\right]=\left[\varphi\left(v_{1}\right), m\right], \quad\left[m, v_{1}\right]=\left[m, \varphi\left(v_{1}\right)\right], \quad \forall v_{1} \in V_{1}, \quad m \in V_{2} .}
\end{aligned}
$$

Proof. For any $v_{1}, v_{2} \in V_{1}, m \in V_{2}$, We just check

$$
\left[\left[v_{1}, v_{2}\right], \alpha_{2}(m)\right]=\left[\alpha_{1}\left(v_{1}\right),\left[v_{2}, m\right]\right]+(-1)^{\left|v_{2}\right||m|}\left[\left[v_{1}, m\right], \alpha_{1}\left(v_{2}\right)\right]
$$

and

$$
\left[v_{1} \cdot v_{2}, \alpha_{2}(m)\right]=\alpha_{1}\left(v_{1}\right) \cdot\left[v_{2}, m\right]+(-1)^{\left|v_{2}\right||m|}\left[v_{1}, m\right] \cdot \alpha_{1}\left(v_{2}\right) .
$$

By the definition of the operations, we have

$$
\begin{aligned}
{\left[\left[v_{1}, v_{2}\right], \alpha_{2}(m)\right] } & =\left[\varphi\left(\left[v_{1}, v_{2}\right]\right), \alpha_{2}(m)\right] \\
& =\left[\left[\varphi\left(v_{1}\right), \varphi\left(v_{2}\right)\right], \alpha_{2}(m)\right] \\
& =\left[\alpha_{2} \varphi\left(v_{1}\right),\left[\varphi\left(v_{2}\right), m\right]\right]+(-1)^{\left|v_{2}\right||m|}\left[\left[\varphi\left(v_{1}\right), m\right], \alpha_{2} \varphi\left(v_{2}\right)\right] \\
& =\left[\varphi \alpha_{1}\left(v_{1}\right),\left[\varphi\left(v_{2}\right), m\right]\right]+(-1)^{\left|v_{2}\right||m|}\left[\left[\varphi\left(v_{1}\right), m\right], \varphi \alpha_{1}\left(v_{2}\right)\right] \\
& =\left[\alpha_{1}\left(v_{1}\right),\left[v_{2}, m\right]\right]+(-1)^{\left|v_{2}\right||m|}\left[\left[v_{1}, m\right], \alpha_{1}\left(v_{2}\right)\right] .
\end{aligned}
$$


and

$$
\begin{aligned}
{\left[v_{1} \cdot v_{2}, \alpha_{2}(m)\right] } & =\left[\varphi\left(v_{1} \cdot v_{2}\right), \alpha_{2}(m)\right] \\
& =\left[\varphi\left(v_{1}\right) \cdot \varphi\left(v_{2}\right), \alpha_{2}(m)\right] \\
& =\alpha_{2} \varphi\left(v_{1}\right) \cdot\left[\varphi\left(v_{2}\right), m\right]+(-1)^{\left|v_{2}\right||m|}\left[\varphi\left(v_{1}\right), m\right] \cdot \alpha_{2} \varphi\left(v_{2}\right) \\
& =\varphi \alpha_{1}\left(v_{1}\right) \cdot\left[v_{2}, m\right]+(-1)^{\left|v_{2}\right||m|}\left[v_{1}, m\right] \cdot \varphi \alpha_{1}\left(v_{2}\right) \\
& =\alpha_{1}\left(v_{1}\right) \cdot\left[v_{2}, m\right]+(-1)^{\left|v_{2}\right||m|}\left[v_{1}, m\right] \cdot \alpha_{1}\left(v_{2}\right) .
\end{aligned}
$$

6.3. Proposition. Let $(V, \circ,[.,],. \alpha)$ be a Hom-Leibniz Poisson superalgebra, then $\left(\operatorname{End}(V), \alpha^{\prime}\right)$ can be endowed with a representation over $V$ by means of the operations

$$
\begin{gathered}
\alpha^{\prime}(f)=\alpha \circ f, \quad(v \cdot f)(a)=v \cdot f(a), \quad(f \cdot v)(a)=(-1)^{|f||a|} f(a) \cdot v, \\
{[v, f](a)=[v, f(a)], \quad[f, v](a)=(-1)^{|v||a|}[f(a), v],}
\end{gathered}
$$

for any $a, v \in V, \quad f \in \operatorname{End}(V)$.

Proof. For any $v_{1}, v_{2} \in V, f \in \operatorname{End}(V)$, We just check

$$
\left[\left[v_{1}, v_{2}\right], \alpha^{\prime}(f)\right]=\left[\alpha\left(v_{1}\right),\left[v_{2}, f\right]\right]+(-1)^{\left|v_{2}\right||f|}\left[\left[v_{1}, f\right], \alpha\left(v_{2}\right)\right]
$$

and

$$
\left[v_{1} \cdot v_{2}, \alpha^{\prime}(f)\right]=\alpha\left(v_{1}\right) \cdot\left[v_{2}, f\right]+(-1)^{\left|v_{2}\right||f|}\left[v_{1}, f\right] \cdot \alpha\left(v_{2}\right)
$$

By the definition of the operations, we have

$$
\begin{aligned}
{\left[\left[v_{1}, v_{2}\right], \alpha^{\prime}(f)\right](a) } & =\left[\left[v_{1}, v_{2}\right], \alpha^{\prime}(f)(a)\right] \\
& =\left[\left[v_{1}, v_{2}\right], \alpha \circ f(a)\right] \\
& =\left[\alpha\left(v_{1}\right),\left[v_{2}, f(a)\right]\right]+(-1)^{\left|v_{2}\right||f|+\left|v_{2}\right||a|}\left[\left[v_{1}, f(a)\right], \alpha\left(v_{2}\right)\right] \\
& =\left[\alpha\left(v_{1}\right),\left[v_{2}, f\right](a)\right]+(-1)^{\left|v_{2}\right||f|+\left|v_{2}\right||a|}\left[\left[v_{1}, f\right](a), \alpha\left(v_{2}\right)\right] \\
& =\left[\alpha\left(v_{1}\right),\left[v_{2}, f\right]\right](a)+(-1)^{\left|v_{2}\right||f|}\left[\left[v_{1}, f\right], \alpha\left(v_{2}\right)\right](a) .
\end{aligned}
$$

Then $\left[\left[v_{1}, v_{2}\right], \alpha^{\prime}(f)\right]=\left[\alpha\left(v_{1}\right),\left[v_{2}, f\right]\right]+(-1)^{\left|v_{2}\right||f|}\left[\left[v_{1}, f\right], \alpha\left(v_{2}\right)\right]$. Since

$$
\begin{aligned}
{\left[v_{1} \cdot v_{2}, \alpha^{\prime}(f)\right](a) } & =\left[v_{1} \cdot v_{2}, \alpha(f(a))\right] \\
& =\alpha\left(v_{1}\right) \cdot\left[v_{2}, f(a)\right]+(-1)^{\left|v_{2}\right||f|+\left|v_{2}\right||a|}\left[v_{1}, f(a)\right] \cdot \alpha\left(v_{2}\right) \\
& =\alpha\left(v_{1}\right) \cdot\left[v_{2}, f\right](a)+(-1)^{\left|v_{2}\right||f|+\left|v_{2}\right||a|}\left[v_{1}, f\right](a) \cdot \alpha\left(v_{2}\right) \\
& =\left(\alpha\left(v_{1}\right) \cdot\left[v_{2}, f\right]\right)(a)+(-1)^{\left|v_{2}\right||f|}\left(\left[v_{1}, f\right] \cdot \alpha\left(v_{2}\right)\right)(a) .
\end{aligned}
$$

We obtain $\left[v_{1} \cdot v_{2}, \alpha^{\prime}(f)\right]=\alpha\left(v_{1}\right) \cdot\left[v_{2}, f\right]+(-1)^{\left|v_{2}\right||f|}\left[v_{1}, f\right] \cdot \alpha\left(v_{2}\right)$.

\section{Acknowledgements}

This work was supported by the National Natural Science Foundation of China (No. 10871057) (No. 11171055), the Natural Science Foundation of Jilin Province (No. 20130101068) and the Fundamental Research Funds for the Central Universities (No. 14QNJJ003). 


\section{References}

[1] Albeverio, S., Ayupov, Sh. A. and Omirov, B. A. On nilpotent and simple Leibniz algebras, Comm. Alg., 33 (1), 159-172, 2005.

[2] Ammar, F., Ayadi, I., Mabrouk, S. and Makhlouf, A. Quadratic color Hom-Lie algebras, arXiv:1204.5155v1, 2012.

[3] Ammar, F. and Makhlouf, A. Hom-Lie superalgebras and Hom-Lie admissible superalgebras, J. Algebra., 324 (7), 1513-1528, 2010.

[4] Ammar, F., Makhlouf, A. and Saadoui, N. Cohomology of Hom-Lie superalgebras and qdeformed Witt superalgebra, Czechoslovak Mathematical Journal, 63(3), 721-761, 2013.

[5] Ayupov, Sh. A. and Omirov, B. A. On Leibniz algebras, Algebra and Operators Theory, $1-12,1998$.

[6] Ayupov, Sh. A. and Omirov, B. A. On 3-dimensional Leibniz algebras, Uzbek Math. J., 1(6), 9-14, 1999.

[7] Ayupov, Sh. A. and Omirov, B. A. On some classes of nilpotent Leibniz algebras, Siberian Math.J., 42 (1), 18-29, 2001.

[8] Casas, J. M. and Datuashoili, T. Noncommutative Leibniz Poisson algebras, Comm. Alg., 34 (7), 2507-2530, 2006.

[9] Casas, J. M. and Pirashvili, T. Algebras with bracket, Manuscripta Math., 119(1), 1-15, 2006.

[10] Casas, J. M. Homology with trivial coefficients and universal central extnesions of algebras with bracket, Comm. Alg., 35 (8), 2431-2449, 2007.

[11] Cuvier, C. Homologie de Leibniz et homologie de hochschild, C.R.Acad. Sci. Paris Se'r. A-B., 313 (12), 569-572, 1991.

[12] Dzhumadil'daev, A. A. Cohomologies of colour Leibniz algebras: Pre-simplicial approach, Lie Theory and its applications in physics III, preceeding of the third international workshop, 124-135, 1999.

[13] Hartwig, J.T., Larsson, D. and Silvesrov, S.D. Deformations of Lie algebras using $\sigma$ derivations, J. Algebra., 295 (2), 314-361, 2006.

[14] Liu, D. and Hu, N. Leibniz superalgebras and central extensions, J. Algebra Appl., 5 (6), 765-780, 2006.

[15] Liu, D. and Hu, N. Steinberg Leibniz algebras and superalgebras, J. Algebra., 283 (1), 199-221, 2005.

[16] Liu, D. and Hu, N. Leibniz central extensions on some infinite dimensional Lie algebras, Comm. Alg., 32 (6), 2385-2405, 2004.

[17] Loday, J. L. Une version non commutative des algèbres de Lie: Les algèbres de Leibniz, Enseign. Math., 39 (1) 269-294, 1993.

[18] Loday, J. L. Cup-product for Leibniz cohomogy and dual Leibniz algebras, Math. Scand., 77 (2), 189-196, 1995.

[19] Loday, J. L. and Pirashvili, T. Universal enveloping algebras of Leibniz algebras and (co)homology, Math. Ann., 296 (1), 139-158, 1993.

[20] Loday, J. L. Dialgebra (co)homology with coefficients, in: Dialgebras and Related Operads, Lecture Notes in Math. 1763 (Springer-Verlag, Berlin, 2001), 67-103.

[21] Makhlouf, A. and Silvestrov, S. D. Notes on formal deformations of Hom-associative and Hom-Lie algebras, Forum Math., 22 (4), 715-739, 2010.

[22] Makhlouf, A. and Silvestrov, S. D. Hom-Lie admissible Hom-coalgebras and Hom-hopf algebra, Generalized Lie Theory in Mathematics, Physics and Beyond, Chapter 17, 189-206, 2009.

[23] Makhlouf, A. and Silvestrov, S. D. Hom-algebras and hom-coalgebras, J. Algebra App., 9 (4), 553-589, 2010.

[24] Makhlouf, A. Hom-dendriform Algebras and Rota-Baxter Hom-algebras, Proc. Int. Conf., in Nankai Series in Pure, Applied Mathematics and Theoretical Physics, (Eds. C. Bai, L. Guo and J-L. Loday), Vol 9, (World Scientific, Singapore, 2012), 147-171.

[25] Sheng, Y. Representations of Hom-Lie algebras, Alg Represent Theory, 5 (6), 1081-1098, 2012 . 
[26] Yau, D. Envelopping algebras of Hom-Lie algebras, J. Gen. Lie Theory Appl., 2(2), 95-108, 2008.

[27] Yuan, L. Hom-Lie color algebra structures, Comm. Alg., 40 (2), 575-592, 2012. 
